DOI 10.37882/2223-2966.2020.10.05

\title{
РАЗРАБОТКА МНОГОКРИТЕРИАЛЬНОЙ ОПТИМИЗАЦИИ ВНЕШНЕТОРГОВОЙ ДЕЯТЕЛЬНОСТИ ПРОМЫШЛЕННОГО ПРЕДПРИЯТИЯ
}

\section{DEVELOPMENT OF MULTI-CRITERIA OPTIMIZATION OF FOREIGN TRADE ACTIVITIES OF AN ENTERPRISE}

V. Bataev

Summary: This article is devoted to the development of multi-criteria optimization of the foreign trade activity of an enterprise, taking into account the management of customs policy instruments in order to ensure the efficiency of the production activity of the enterprise as a whole. The article presents problem-oriented mathematical models of multivariate assessment of the efficiency of the enterprise in the process of implementing foreign economic activity, built on the basis of the CCR and Super-efficiency models of the DEA method. An assessment of the economic efficiency of the implementation of foreign economic activity of JSC «Arkonik SMZ» is based on the results of the analysis of the carried out calculations.

Keywords: foreign economic activity, customs operations, Data Envelopment Analysis (DEA), input and output parameters, comparative efficiency assessment.

\section{Введение}

$\mathrm{P}$ оль внешнеэкономической деятельности (ВЭД), охватывающей внешнюю торговлю с третьими странами, особенно высока в условиях рыночной экономики. Расширение внешнеэкономических связей отечественных предприятий способствует интеграции Российской Федерации в мировую экономику.

Для многих отечественных предприятий внешнеэкономические операции являются неотъемлемой частью всей финансово-хозяйственной деятельности. Однако, ведение внешней торговли товарами характеризуется наличием административных барьеров, сдерживающих темпы товарооборота. При этом государственное регулирование внешнеторговой деятельности создаёт для участников ВЭД неудобства при совершении таможенных операций, влечёт дополнительные незапланированные непроизводственные издержки.

В то же время анализ права Евразийского экономического союза и нормативных актов национального уровня $[1,2,3,4]$ показывает, что при внедрении системного подхода к осуществлению внешнеторговой деятельно-

\author{
Батаев Вячеслав Викторович \\ Аспирант, Самарский государственный технический \\ университет \\ bataev.63@mail.ru
}

Аннотация: Статья посвящена разработке многокритериальной оптимизации внешнеторговой деятельности промышленного предприятия с учетом управления инструментами таможенной политики в целях обеспечения эффективности производственной деятельности предприятия в целом. В статье приведены проблемно-ориентированные математические модели многофакторной оценки эффективности функционирования предприятия в процессе реализации внешнеэкономической деятельности, построенные на основе CCR и Super-efficiency моделей DEA-метода. По результатам анализа проведённых расчётов дана оценка экономической эффективности осуществления внешнеторговой деятельности АО «Арконик СМЗ».

Ключевые слова: внешнеэкономическая деятельность, совершение таможенных операций, сравнительная оценка эффективности.

сти появляются возможности сокращения непроизводственных издержек, связанных с соблюдением установленных запретов и ограничений.

\section{Постановка задачи и выбор объектов сравнения}

В качестве объекта системного анализа в диссертационной работе выбрано промышленное предприятие, осуществляющее внешнеэкономическую деятельность во взаимодействии с таможенными органами Российской Федерации. Объект анализа рассмотрен как единая система, так как он обладает основными системными свойствами: целостностью и делимостью, наличием существенных устойчивых связей между элементами системы, организацией и иерархической структурой системы, интегративными качествами.

Рассмотренная система состоит из двух подсистем: системы промышленного предприятия и системы таможенных органов.

Для сравнительного анализа эффективности выбраны варианты совершения таможенных операций участником ВЭД во взаимодействии с таможенными органами. 
Выбранные варианты представляют собой различные сочетания таких действий, как: подача, принятие и регистрация или авторегистрация таможенной декларации; форматно-логический контроль сведений, заявленных в таможенной декларации; запрос дополнительных документов и сведений; проведение таможенного досмотра и иных форм таможенного контроля; получение и представление для таможенных целей разрешительных документов; принятие решения о выпуске или отказе в выпуске декларируемых товаров.

Основной задачей исследования является разработка многокритериальной оптимизации внешнеторговой деятельности промышленного предприятия для обеспечения максимальной эффективности производственной деятельности предприятия в целом. Для этих целей проведено сравнение выбранных объектов и определение наиболее оптимальных и экономически эффективных вариантов совершения таможенных операций.

На основе проведенного анализа операций по экспорту готовой продукции собственного производства $\mathrm{AO}$ «Арконик СМЗ» выявлен $\mathrm{N}=21$ вариант совершения таможенных операций, определены характеристики этих операций, которые явились основой для формирования критериев сравнительной эффективности таможенных операций.

\section{Применение метола Data Envelopment Analysis (DEA) в качестве математического аппарата Аля провеАения расчётов.}

Для оценки эффективности осуществления ВЭД промышленным предприятием использован метод DEA, являющийся математическим методом, позволяющим производить оценку и сравнение деятельности или эффективности различных систем между собой.

Модель сравнительной оценки эффективности операций ВЭД при экспорте готовой продукции собственного производства согласно базовой постановке DEAметода, показана на рисунке 1.

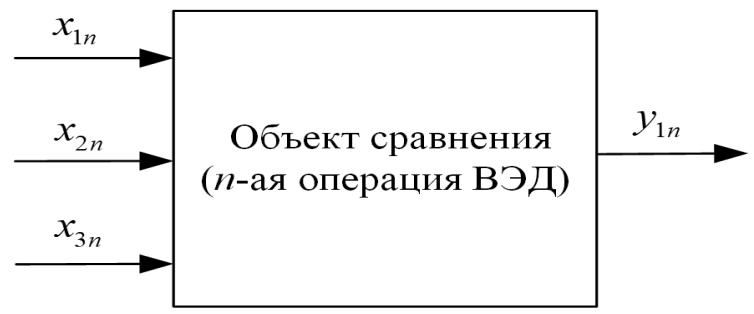

Рис.1. Модель сравнительной оценки эффективности операций ВЭД при экспорте готовой продукции собственного производства

Входными параметрами $x_{m n}, m=\overline{1,3}, n=\overline{1,21}$ модели сравнительной оценки эффективности таможенных операций при экспорте продукции являются следующие критерии:

- $x_{1 n}$ - трудозатраты предприятия, характеризующие работу, выполняемую сотрудниками предприятия за календарный год, и определяющиеся по формуле:

$$
x_{1 n}=K_{n} \cdot t_{n \text { [чел.•час/год], }}
$$

где $K_{n}$ - общее годовое количество товарных партий, экспортированных с применением $n$-ой таможенной операции [шт./год];

$t_{n}$ - трудозатраты сотрудников предприятия на оформление товарных партий по n-ой операции ВЭД [чел.ччас/шт.];

$n$ - порядковый номер операции ВЭД при экспорте готовой продукции собственного производства, $n=\overline{1, N}$.

- $x_{2 n}$ - общие затраты предприятия на экспортные операции, включающие трудозатраты сотрудников предприятия, связанные с декларированием, выраженные в денежном выражении, и обязательные затраты при таможенном декларировании (получение лицензий ФСТЭК), и определяются по формуле:

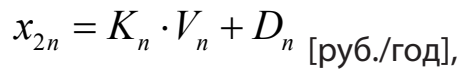

где $V_{n}$ - трудозатраты сотрудников предприятия в денежном выражении на оформление декларации и других связанных работ по $n$-ой таможенной операции [руб./шт.];

$D_{n}$ - обязательные затраты на таможенное декларирование по n-ой таможенной операции [руб./год].

- $x_{3 n}$ - необязательные сопутствующие затраты предприятия при экспорте товаров, связанные с получением идентификационных заключений для целей экспортного контроля, простоем транспортных средств и штрафными санкциями за нарушение таможенных правил, и определяются путём суммирования всех необязательных затрат по каждой $n$-ой таможенной операции за год [руб./год].

Выходным параметром $y_{1 n}, n=\overline{1,21}$ модели сравнительной оценки эффективности таможенных операций при экспорте готовой продукции собственного производства является отношение прибыли предприятия от ВЭД к срокам совершения $n$-ой операции от регистрации ДТ до выпуска товаров за календарный год, который определяется по формуле:

$$
y_{1 n}=\frac{P_{n}}{T_{1 n}} \text { [руб./час], }
$$

где $P_{n}$ - годовая прибыль предприятия от реализации продукции, экспортированной с применением $n$-ой та- 
моженной операции [руб./год];

$T_{1 n}$ - время, затраченное на совершение $n$-ой таможенной операции [час/год].

Исходные данные для модели сравнительной оценки эффективности таможенных операций представлены в таблицах 1 - 3 для 2017-2019 годов, соответственно.

Формулировка ЗМП для определения сравнительной оценки $E_{n}$ эффективности таможенных операций на основе базовой CCR модели DEA-метода может быть представлена для $n$-ой таможенной операции в виде:

$$
\begin{gathered}
E_{n}=\frac{u_{1 n} y_{1 n}}{\sum_{i=1}^{3} v_{i n} x_{i n}} \rightarrow \max _{(U, V) \in G} ; \\
\frac{u_{1 n} y_{1 n}}{\sum_{i=1}^{3} v_{i n} x_{i n}} \leq 1, \forall\left(n=\overline{1,21} ; u_{1 n} \geq 0 ; v_{i n} \geq 0, i=\overline{1,3}\right),
\end{gathered}
$$

где $U=u_{1 n} ; V=\left(v_{i n}\right), i=\overline{1,3}$ - векторы весовых коэффициентов для $n$-го объекта в анализируемой группе.

Задача (4)-(5) при $n=\overline{1,21}$ представляет собой 21 ЗМП, решение которых позволяет определить относительные значения оценок $E_{n^{\prime}} n=\overline{1,21}$ для всех $N$ таможенных операций в анализируемой группе на основе базовой CCR модели DEA-метода. Оценки $E_{n}$ распределены на единичном интервале $[0,1]$. Таможенные операции с оценкой равной $E_{n}=1$ считаются наиболее эффективными в анализируемой группе.

В целях определения самой эффективной таможенной операции формулируются ЗМП для определения сравнительной оценки $S E_{\text {н }}$ на основе модели Superefficiency DEA-метода, которая для n-ой таможенной операции может быть представлена в виде:

$$
S E_{n}=\frac{\bar{u}_{1 n} y_{1 n}}{\sum_{i=1}^{3} \bar{v}_{i n} x_{i n}} \rightarrow \max _{(\bar{U}, \bar{V}) \in \bar{G}}
$$

$\frac{\bar{u}_{1 n} y_{1 n}}{\sum_{i=1}^{3} \bar{v}_{i n} x_{i n}} \leq 1, \forall\left(n=\overline{1,21} ; n \neq k ; \bar{u}_{1 n} \geq 0 ; \bar{v}_{i n} \geq 0, i=\overline{1,3}\right)$

где $\bar{U}=\bar{u}_{1 n} ; \bar{V}=\left(\bar{v}_{i n}\right), i=\overline{1,3}$ - векторы весовых коэ $\phi$ фициентов для каждой операции, которые за исключением весовых коэффициентов $\bar{u}_{1 k}$ и $\bar{v}_{i k}, i=\overline{1,3}$ являются неизвестными;

$\bar{u}_{1 k}$ - весовой коэффициент, полученный для выходной характеристики $k$-ой таможенной операции со значением оценок эффективности $E_{k}=1$, рассчитанным на основе решения задачи (4)-(5);

$\bar{v}_{i k}, i=\overline{1,3}$ - весовые коэффициенты, полученные для входных параметров $k$-ой таможенной операции со значением оценок эффективности $E_{k}=1$, рассчитанным на основе решения задачи (4)-(5);

$k$ - порядковый номер эффективной таможенной операции со значением оценки эффективности $E_{k}=1$ в анализируемой группе, которые исключаются из решения ЗМП в форме (6)-(7).

Задача (6)-(7) при $n=\overline{1,21}$ в (6) представляет собой 21 ЗМП, решение которых позволило определить оценки эффективности $S E_{n^{\prime}} n=\overline{1,21}$ для $n$-ой таможенной операций по модели Super-efficiency DEA-метода. Оценка $S E_{n}$ распределена на интервале $[0, \square)$.

Задача (6)-(7) при $n=\overline{1,21}$ в (6) представляет собой 21 ЗМП, решение которых позволило определить относительные значения оценок эффективности $S E_{n^{\prime}} n=\overline{1,21}$ таможенных операций при экспорте продукции на основе модели Super-efficiency DEA-метода.

1.

\section{Анализ полученных результатов (вхо $\Delta$ ные и выходной параметры).}

Анализ полученных результатов показал, что в 2018 и 2019 году лучшей таможенной операцией явилась операция №1, сопровождавшаяся автоматической регистрацией ДТ и автоматическим выпуском товаров, с оценками эффективности $S E_{1}^{2018}=86420433$ и $S E_{1}^{2019}=3.019$. При этом величина оценки эффективности за 2018 год операции №1 свидетельствует о подавляющем превосходстве над эффективностью остальных таможенных операций.

В 2017 году самой эффективной стала операция №10 с оценкой $S E_{10}^{2017}=10.698$. Данная таможенная операция характеризуется отказом в регистрации ДТ и возвратом на предыдущий уровень. Эффективной данную операцию делают нормативно установленные минимальные сроки для отказа в регистрации ДТ (не более 1 часа) и подача новой ДТ с учётом выявленных и устранённых несоответствий. Не имеющая изъянов вновь подаваемая ДТ обеспечивает выпуск товаров в минимально возможные нормативно установленные сроки.

Самой неэффективной операцией явилась операция №15 с оценками $S E_{15}^{2017}=0.007, S E_{15}^{2018}=0.002, S E_{15}^{2019}=0.002$, соответственно, за период с 2017 по 2019 год. Неэффективной данную операцию делает выявление в процессе таможенного декларирования и таможенного контроля контролируемого статуса экспортируемой продукции. При этом помимо затрат на получение разрешительного документа (лицензии ФСТЭК) декларант (промышленное предприятие) несёт непроизводственные издержки, связанные с простоем транспортного средства и штрафными санкциями со стороны таможенных органов в со- 
Таблица 1.

Исходные данные для модели сравнительной оценки эффективности операций

ВЭД при экспорте продукции за 2017 год

\begin{tabular}{|c|c|c|c|c|}
\hline \multirow{2}{*}{$\begin{array}{c}\text { № oперации ВЭД } \\
n=2,20\end{array}$} & \multicolumn{3}{|c|}{ Входные параметры $m=\overline{1,3}$} & \multirow{2}{*}{$\frac{\text { Выходной параметр }}{y_{1 n}}$} \\
\hline & $x_{1 n}$ & $x_{2 n}$ & $x_{3 n}$ & \\
\hline 2 & 3505.06 & 1452914 & 0 & 124183.98 \\
\hline 3 & 40.25 & 38716 & 24000 & 81.50 \\
\hline 4 & 927.32 & 605132 & 120000 & 42846.11 \\
\hline 5 & 31.25 & 27578 & 24000 & 14758.45 \\
\hline 6 & 720.92 & 540135 & 1512000 & 22947.92 \\
\hline 7 & 15.50 & 5997 & 60000 & 18703.90 \\
\hline 8 & 7.83 & 10363 & 134010 & 1053.97 \\
\hline 9 & 10.20 & 11351 & 163822 & 790.41 \\
\hline 10 & 153.90 & 53339 & 0 & 48773.82 \\
\hline 11 & 2213.74 & 963442 & 84000 & 34535.78 \\
\hline 12 & 55.80 & 37640 & 60000 & 26522.01 \\
\hline 13 & 468.06 & 204602 & 1044000 & 31330.87 \\
\hline 14 & 53.90 & 45877 & 24000 & 14647.91 \\
\hline 15 & 242.73 & 99332 & 3460352 & 1907.29 \\
\hline 16 & 6.20 & 11692 & 155094 & 1053.41 \\
\hline 17 & 106.56 & 66069 & 427551 & 2715.93 \\
\hline 18 & 19.76 & 15558 & 78760 & 3523.79 \\
\hline 19 & 13.83 & 5705 & 121722 & 873.88 \\
\hline 20 & 44.64 & 40810 & 534004 & 758.98 \\
\hline
\end{tabular}

Таблица 2

Исходные данные для модели сравнительной оценки эффективности операций ВЭД при экспорте продукции за 2018 год

\begin{tabular}{|c|c|c|c|c|}
\hline \multirow{2}{*}{$\begin{array}{c}\text { № onерации ВЭД } \\
n=1,20\end{array}$} & \multicolumn{3}{|c|}{ Входные параметры $m=\overline{1,3}$} & \multirow{2}{*}{$\frac{\text { Выходной параметр }}{y_{1 n}}$} \\
\hline & $x_{1 n}$ & $x_{2 n}$ & $x_{3 n}$ & \\
\hline 1 & 733.46 & 288338.75 & 0 & 2043100.70 \\
\hline 2 & 3890.38 & 1686891.25 & 72000 & 170218.13 \\
\hline 3 & 17.25 & 14449.50 & 24000 & 15428.21 \\
\hline 4 & 985.52 & 853048.50 & 84000 & 84462.05 \\
\hline 5 & 12.50 & 12531.00 & 24597 & 20551.23 \\
\hline 6 & 548.76 & 487378.50 & 2988894 & 32439.38 \\
\hline 7 & 31.00 & 26994.00 & 96597 & 23202.48 \\
\hline 8 & 7.83 & 10363.25 & 125477 & 1533.93 \\
\hline 10 & 69.00 & 46206.00 & 60000 & 70717.93 \\
\hline 11 & 1566.47 & 792880.75 & 96000 & 48107.84 \\
\hline 12 & 18.60 & 15046.50 & 61791 & 29746.85 \\
\hline 13 & 371.22 & 334770.75 & 1313193 & 32345.32 \\
\hline 15 & 454.14 & 935846.50 & 4119876 & 2370.55 \\
\hline 16 & 12.40 & 30884 & 365514 & 530.90 \\
\hline 17 & 79.92 & 62676.75 & 265370 & 5537 \\
\hline 18 & 39.52 & 31115.00 & 283496 & 2733.70 \\
\hline 20 & 14.88 & 13205.25 & 178962 & 134.38 \\
\hline
\end{tabular}


Таблица 3.

Исходные данные для модели сравнительной оценки эффективности операций

ВЭД при экспорте продукции за 2019 год

\begin{tabular}{|c|c|c|c|c|}
\hline \multirow{2}{*}{$\begin{array}{l}\text { № onерации ВЭД } \\
n=1,21\end{array}$} & \multicolumn{3}{|c|}{ Входные параметры $m=\overline{1,3}$} & \multirow{2}{*}{$\frac{\text { Выходной параметр }}{y_{1 n}}$} \\
\hline & $x_{1 n}$ & $x_{2 n}$ & $x_{3 n}$ & \\
\hline 1 & 4681.30 & 1877819 & 120000 & 1848134.95 \\
\hline 2 & 5708.82 & 2514259 & 192000 & 103812.42 \\
\hline 3 & 11.50 & 12133 & 12000 & 28809.98 \\
\hline 4 & 737.20 & 1055273 & 144000 & 31918.41 \\
\hline 5 & 18.75 & 22547 & 48873 & 19962.91 \\
\hline 6 & 489.58 & 289009 & 682500 & 12487.96 \\
\hline 7 & 7.75 & 2999 & 24291 & 11544.63 \\
\hline 8 & 23.49 & 31090 & 218675 & 4086.23 \\
\hline 9 & 10.20 & 11692 & 157838 & 1002.65 \\
\hline 10 & 62.70 & 44231 & 60000 & 306120.71 \\
\hline 11 & 306.40 & 169940 & 72000 & 36721.56 \\
\hline 12 & 12.40 & 20031 & 36291 & 36364.65 \\
\hline 13 & 188.30 & 97311 & 420000 & 13296.00 \\
\hline 14 & 7.70 & 3340 & 24291 & 16712.07 \\
\hline 15 & 399.33 & 178417 & 3122132 & 2778.44 \\
\hline 16 & 6.20 & 11692 & 171424 & 1001.76 \\
\hline 17 & 97.68 & 62438 & 313725 & 5972.41 \\
\hline 18 & 19.76 & 15558 & 93342 & 1392.85 \\
\hline 21 & 12.20 & 12517 & 95938 & 2572.83 \\
\hline
\end{tabular}

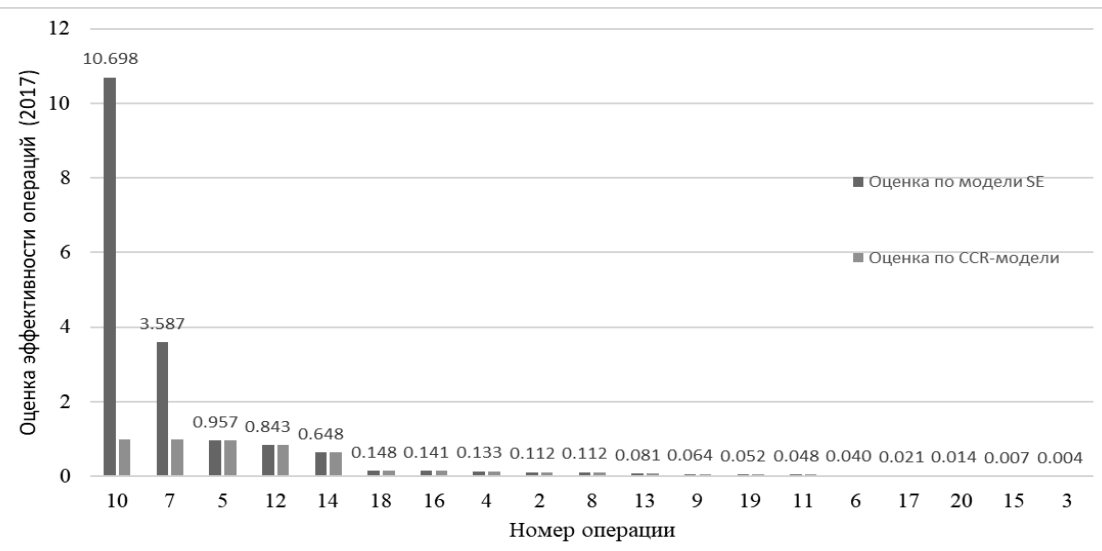

Рис. 2. Ранжировка оценок эффективности таможенных операций за 2017 год

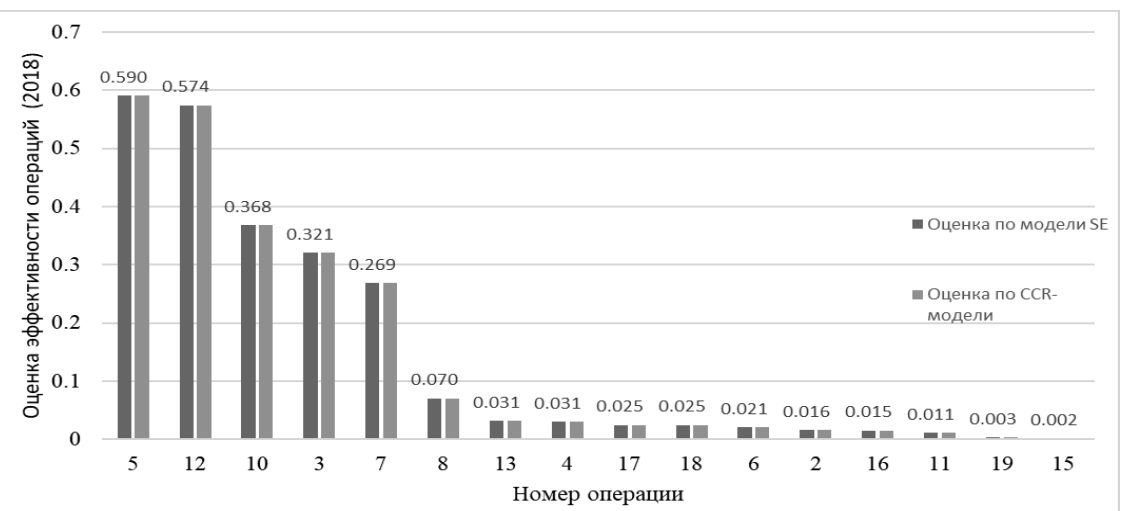

Рис. 3. Ранжировка оценок эффективности таможенных операций за 2018 год 


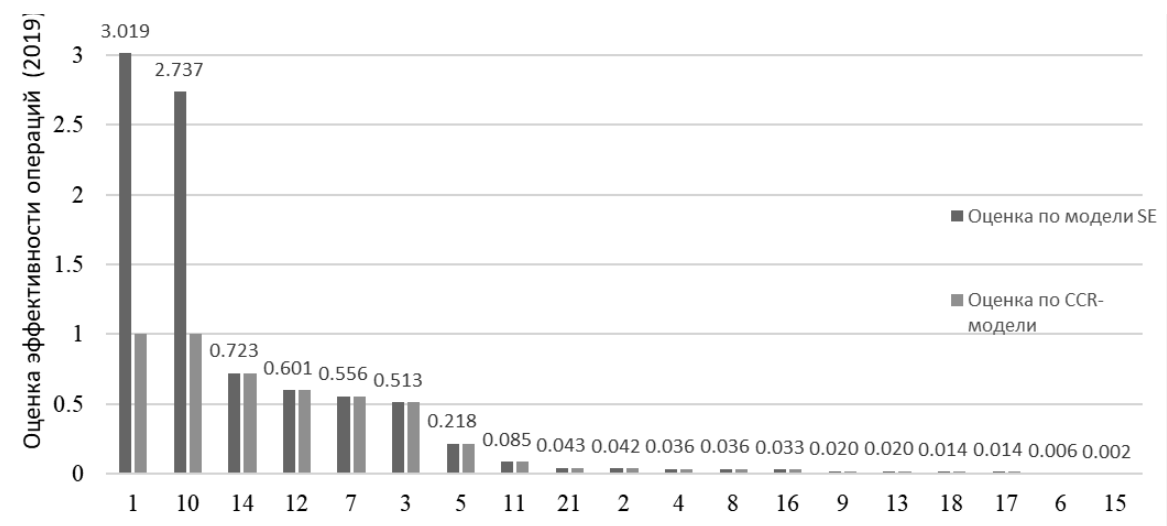

Рис. 4. Ранжировка оценок эффективности таможенных операций за 2019 год

ответствии со статьёй 16.3 КоАП России.

На рисунках 2 - 4 представлены ранжированные оценки эффективности таможенных операций за 2017 2019 годы, соответственно.

Расчёты, проведённые с помощью DEA-метода, и анализ полученных результатов выявили низкую экономическую эффективность тех вариантов совершения таможенных операций, которые сопровождаются представлением для таможенных целей разрешительных документов. А варианты совершения таможенных операций, связанные с проведением таможенного досмотра или отказом в выпуске товаров с последующим повторным декларированием, по результатам проведённых расчётов и анализа полученных данных оказались для предприятия менее затратными, чем те, которые обременены получением разрешительных документов.

Применение системного подхода промышленным предприятием к выстраиванию собственной таможенной политики и тщательной проработки внешнеторговых сделок на каждом из этапов их реализации позволит снизить количество получаемых разрешительных документов, а в большинстве случаев исключить необходимость их оформления.

\section{Зак^ючение}

Взятый за основу описанный методологический подход, а также выбранный и обоснованный способ решения позволили провести многофакторную оценку эффективности внешнеэкономической деятельности промышленного предприятия с помощью методики сравнительного анализа, который базируется на использовании DEA-метода. Данный метод до настоящего времени не применялся для целей определения сравнительной эффективности и иерархического построения вариантов совершения таможенных операций в анализируемой подсистеме.

Проблемно-ориентированные математические модели многофакторной оценки эффективности функционирования предприятия в процессе реализации ВЭД, построенные на основе CCR и Super-efficiency моделей метода анализа оболочки данных или Data Envelopment Analysis (DEA), впервые применены к рассматриваемому объекту исследования.

Разработанные на основе DEA-метода процедуры многокритериальной оптимизации системы взаимодействия предприятия с таможенными органами, в которой инструменты таможенной политики рассматриваются как управляющие факторы, используются для повышения эффективности ВЭД предприятия.

ЛИТЕРАТУРА

1. Таможенный кодекс Евразийского экономического союза.

2. Решение Евразийской экономической комиссии от 25.12.2012 №294 «0 Положении о порядке ввоза на таможенную территорию Таможенного союза продукции (товаров), в отношении которой устанавливаются обязательные требования в рамках Таможенного союза».

3. Федеральный закон от 03.08.2018 №289-Ф3 «0 таможенном регулировании в Российской Федерации и о внесении изменений в отдельные законодательные акты Российской Федерации».

4. Федеральный закон от 18.07.1999 №183-Ф3 «06 экспортном контроле».

5. Charnes, A.Measuring the efficience of Decision Making Units [Text] / A.Charnes, W.W.Cooper, E.Rhodes // European journal of operational research. - 1978.Vol. 2.-P. 429-444. 
6. Кривоножко В.Е., Пропой А.И., Сеньков Р.В., Родченков И.В., Анохин П.М. Анализ эффективности функционирования сложных систем // Автоматизация проектирования.1999. №1. С. 2-7.

7. Кривоножко В.Е., Лычев А.В. Анализ деятельности сложных социально-экономических систем // М.: Издательский отдел факультета ВМиК МГУ. МАКС Пресс. 2010. 208c.

8. Афонин П.Н. Системный анализ и управление в таможенном деле: учебное пособие // СПб.:Интермедиа. 2015. 370 c.

9. Макрусев В.В. Системный анализ в таможенном деле: учебное пособие // М.-Берлин. Директ-Медиа. 2015. 471 с.

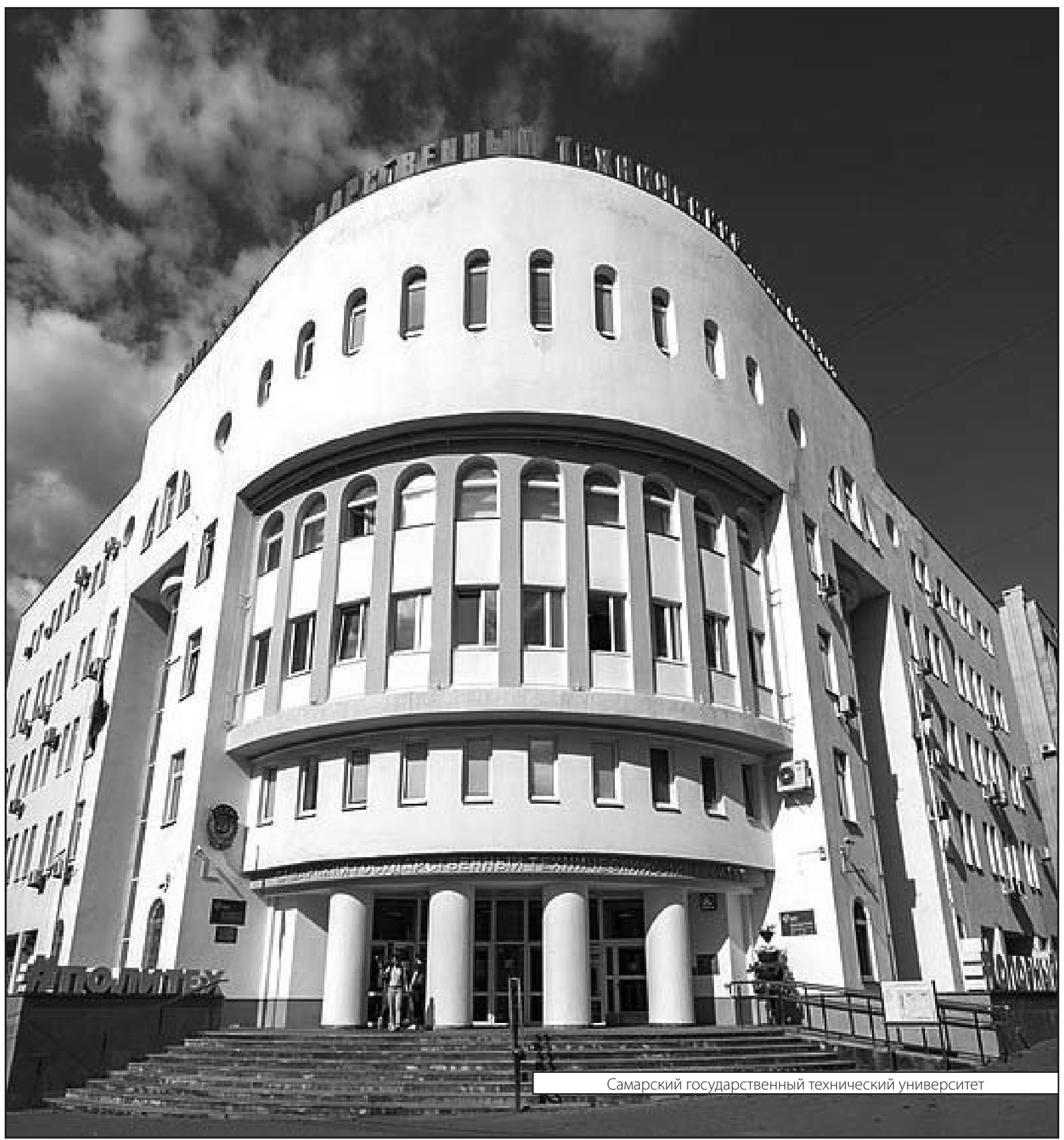

